\title{
Calcium Sparks in Cardiac Cells in Silico
}

\author{
Ryvkin A.M.1,2 Markov N.S. ${ }^{2}$ \\ 1 Institute of Immunology and Physiology UrB RAS, Ekaterinburg, Russia; \\ 2 Ural Federal University, , Ekaterinburg, Russia; shatzkarts@gmail.com \\ * Correspondence: a.ryvkin@iip.uran.ru
}

\begin{abstract}
We simulate elementary calcium release events (sparks) in a single calcium release unit in ventricular myocyte. Previously developed and tested electron-conformational model of the stochastic dynamics of RyR-channels is integrated to the calcium dynamics model in the cardiac cell. This approach allows to observe RyRs opening/closing in details on the macromolecular level during the calcium dynamics course. We simulate calcium diffusion in the dyadic space and "domino-like" RyR's activation during the so-called "calcium induced-calcium release process". $\mathrm{Ca}^{2+}$ sparks initiation, spread and termination are investigated in the computer experiments. Sparks' initiation and termination rate dependence on the $\mathrm{Ca}^{2+}$ diffusion velocity is observed. We show that sarcoplasmic reticulum lumen local depletion and RyR's stochastic attrition could be the reasons of $\mathrm{Ca}^{2+}$ spark termination.
\end{abstract}

Key Words: Calcium signaling, calcium release, sparks, ventricular cardiac myocytes, RyR-channels.

\section{Introduction}

In the cardiac cells action potential generation and contraction is a result of a complex process of triggered calcium release from sarcoplasmic reticulum. Elementary events of $\mathrm{Ca}^{2+}$ release from the sarcoplasmic reticulum (SR) through ryanodine receptors (RyR-channels or RyRs) are called calcium sparks [1] which are initiated by $\mathrm{Ca}^{2+}$ influx to the cell through L-type channels rising $\mathrm{Ca}^{2+}$ concentration enough to activate RyR-channels. This phenomenon is called Calcium Induced Calcium Release (CICR) [2] and it plays a major role in the calcium signaling in cardiac and muscle cells. A detailed study of calcium sparks is essential for understanding the role of calcium signaling in cardiac cells [3-5].

Without the extracellular signal spontaneous calcium sparks can be a reason of the action potential generation automaticity in cardiac pacemaker cells, however in ventricular cardiac cells they may trigger calcium waves and can be a reason of extrasystolic contraction and act as a root of arrhythmias[6].

There is an urgent need of an adequate mathematical model which can be used to simulate calcium sparks and can describe RyR's activation process in details. Here we introduce computational model of spark dynamics which includes Electron-Conformation Model (ECM) [7-10] (a detailed theory of RyR-channels stochastic dynamics) and a theory of calcium and calcium buffers (calmodulin and calsequestrin) dynamics in the calcium release units in cardiac cells. ECM is a simple and biophysically reasonable theory of RyR's stochastic dynamics describes RyR behavior in terms of open and closed electronic state. In addition to the fast electronic degree of freedom, RyR channels are characterized by the slow classical conformational coordinate, which specifies the RyR channel conductance. The RyR gating implies a conformational Langevin dynamics, $\mathrm{Ca}^{2+}$-induced electronic transitions, quantum tunneling and thermal transitions $[8,9]$.

The problem of the calcium diffusion process in the compartments of the cardiac cells traditionally is solved by finite elements method due to the complex geometry of the calcium release system $[2,11]$. For the quick computations we use finite differences method to solve the 2D problem of $\mathrm{Ca}^{2+}$ diffusion in the dyadic space of RU which is described by the standard diffusion equation. The main advantage of our approach is a robustness of the computer simulations.

In the current paper we present our computer experiments results of calcium sparks initiationspread-termination process. 
The reason of $\mathrm{Ca}^{2+}$ spark termination is still a subject of discussion. No single mechanism is able to explain the process of CICR extinguishing. There are different points of view on this problem. First: local $\mathrm{Ca}^{2+}$ depletion. Calcium release reduces $\mathrm{Ca}^{2+}$ lumen concentration thereby drops $\mathrm{RyR}^{\prime}$ s activation probability from the lumen side [1, 2]. Second: RyR's stochastic attrition. Random closing of RyRchannels is able to stop spark spreading process [2]. One of the main aims of our research is to prove these ideas.

\section{MATHEMATICAL MODEL OF CALCIUM RELEASE}

\section{Model geometry}

Calcium release system in cardiac cells (pic.1a) consists of sarcoplasmic reticulum network and SR terminal cisternae (lumens) which are located near t-tubule (small tubules which run transversely through a muscle fiber and through which electrical impulses are transmitted from the sarcoplasm to the fiber's interior).

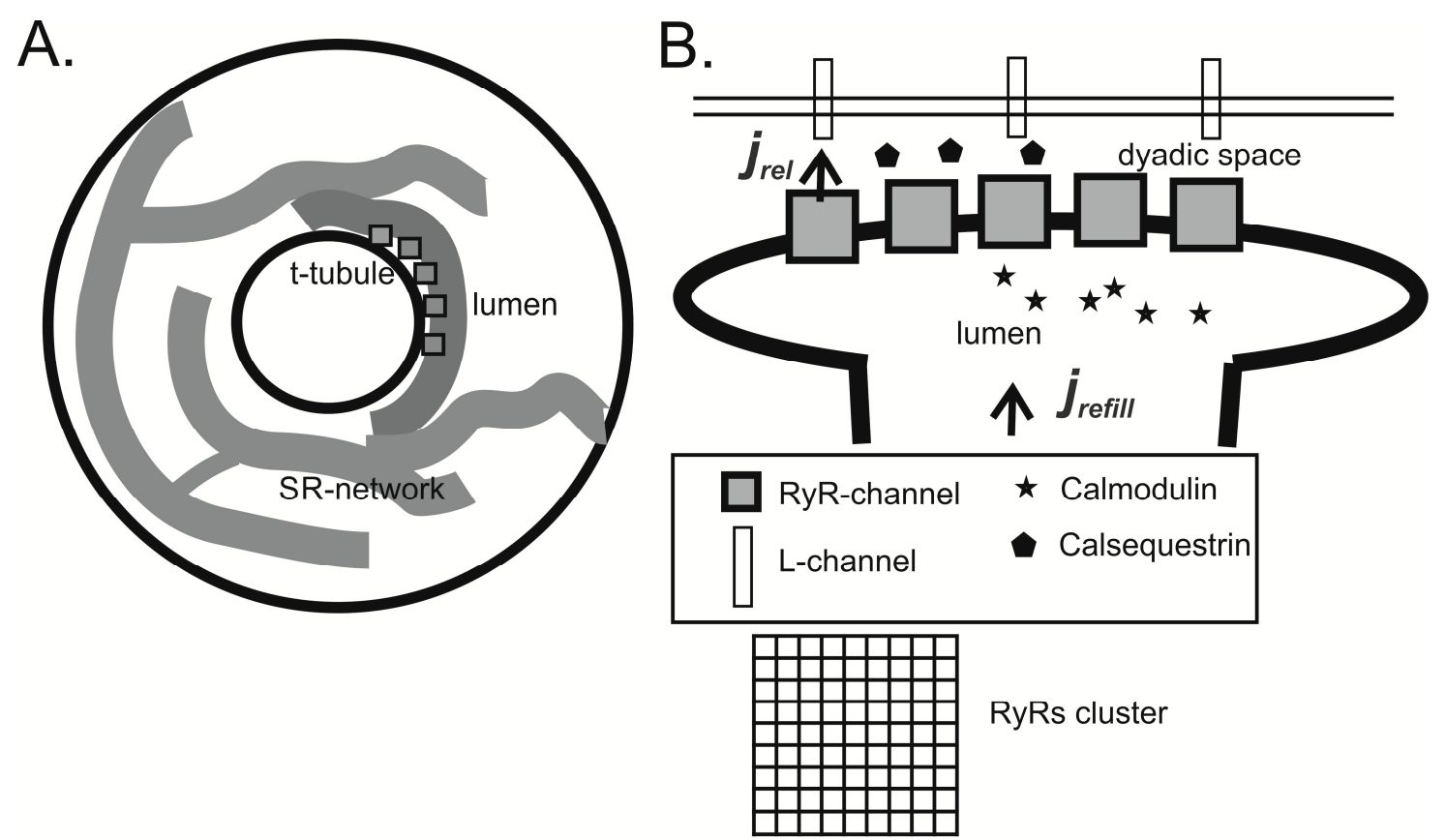

Pic. 1. Illustration of the model geometry. a) Cross-section of a cylindrical model with a t-tubule in the center. b) A single $\mathrm{Ca}^{2+}$-release unit, containing SR-lumen (jSR, junctional sarcoplasmic reticulum), a cluster (9x9) RyR-channels and a dyadic space. RyR's square cluster is located in jSR membrane. T-tubule membrane contains potential activated L-type calcium channels. Released from jSR calcium diffuses across the dyadic space in xy-directions. Calcium buffers: calmodulin (CM) is located in jSR and calsequestrin is in the dyadic space. jSR is connected with SR-network and is refilled by a constant flux $j_{\text {refill }}$.

\section{Model equations}

$\mathrm{Ca}^{2+}$ concentrations in the release unit (RU) compartments and $\mathrm{Ca}^{2+}$ buffers dynamics are described by the following system: 


$$
\begin{aligned}
& \frac{d C a_{j S R}}{d t}=j_{r e f i l l}-j_{r e l}-C Q_{t o t} \cdot \frac{d f_{C Q}}{d t} \\
& \frac{d f_{C Q}}{d t}=k_{f C Q} C a_{j S R}\left(1-f_{C Q}\right)-k_{b C Q} f_{C Q} \\
& \frac{d C a_{S S}}{d t}=\frac{V_{j S R}}{V_{S S}} j_{r e l}-C M_{t o t} \frac{d f_{C M}}{d t} \\
& \frac{d f_{C M}}{d t}=k_{f C M} C a_{S S}\left(1-f_{C M}\right)-k_{b C M} f_{C M}
\end{aligned}
$$

where $C a_{j s R}$ is a lumen concentration, Cass is an average dyadic space concentration, $f_{C Q}$ is a calsequestrin concentration, $f_{C M}$ is a calmodulin concentration, CQtot: total calsequestrin concentration. CMtot: total calmodulin concentration. $k_{f C M}, k_{f C Q}-$ calmodulin and calsequestrin association constants, . $k_{b C M}, k_{b C Q}-$ calmodulin and calsequestrin dissociation constants, $j_{r e f i l l}-$ constant $\mathrm{Ca}^{2+}$ flux to the SR lumen, $j_{\text {rel }}$ - total release flux from the lumen through all opened RyR-channels which equals $j_{\text {rel }}=N_{\text {open }} \cdot k_{\text {rel }}\left(C a_{j S R}-C a_{S S}\right)$, where $N_{\text {open }}$ is a number of open RyRs.

Calcium dynamics across the dyadic space is described in 2D case by the diffusion equation:

$$
\frac{\partial C a_{S S_{\_} m e s h}}{\partial t}=d \cdot\left(\frac{\partial^{2} C a_{S S_{\text {_mesh }}}}{\partial x^{2}}+\frac{\partial^{2} C a_{S S_{\_} m e s h}}{\partial y^{2}}\right)+f_{\text {rel }+ \text { buff }} \text {, }
$$

where Cass_mesh is a local dyadic space $\mathrm{Ca}^{2+}$ concentration, $d$ is a diffusion coefficient, $f_{\text {reltbuff }}$ is a nonlinear term, describing $\mathrm{Ca}^{2+}$ release to the dyadic space and calcium buffers influence.

RyR's transition probability in the Electron-Conformational model is divided into fast electronic transition probability and fast tunneling probability. Electronic transition probability depends on local $\mathrm{Ca}^{2+}$ concentration near each RyR and assumed as "threshold-like":

$P_{\text {elect }}\left(C a_{S S}\right)=\left\{\begin{array}{l}0, C a_{S S}<C a_{S S_{-} \text {crit }} \\ \alpha \cdot C a_{S S}, C a_{S S} \geq C a_{S S_{\text {_c }} \text { crit }}\end{array}\right.$

where Cass_crit is the threshold concentration of $\mathrm{Ca}^{2+}$ in the dyadic space, which defines the beginning of the electronic activation of the channels.

Tunneling probability depends on the lumen concentration $P_{t u n}=P_{t u n}\left(C a_{j s R}\right)$ and the tunneling from the closed to the open state occurs for $C a_{j S R}>K_{c a}$, as well as for $C a_{j S R}<K_{c a}$ transitions from the open to the closed state take place, where $K_{c a}$ is a critical concentration of calcium ions in the SR lumen at which minimums of conformational potential are balanced. For details see references [7-9].

\section{Computational Methods}

For the numerical solution of (2) a rather simple implicit Euler numerical scheme of the finite differences method was used. To solve the matrix system of equations taking into account the question of the problem scalability, the PETSc library is proposed (https://www.mcs.anl.gov/petsc/) for C++. This package provides for use a set of distributed data structures like parallel matrices and vectors, as well as a set of the most efficient parallel solvers of the linear equations systems and preconditioners.

Numerical simulation is formulated by considering integers $m_{1}, m_{2}$ such that $h_{x}=D_{x} / m_{1}, h_{y}=D_{y} / m_{2}$ are characteristics of space grid. Orthogonal uniform computational mesh includes $m_{1} \times m_{2}$ numerical nodes, $h_{x}$ and $h_{y}$ are steps by space in orthogonal directions. Time grid is defined by real numbers $\tau$ and $T$ where $\tau$ is a time step interval and $N=T / \tau$ is a number of conducted time steps. Variable $u^{n_{i, j}}$ is a value of unknown concentration Cass $\left(x_{i}, y_{j}, t_{n}\right)$ in subspace.

Approximation of () leads to a following numerical scheme:

$$
u_{i, j}^{n}+\frac{\tau}{h_{x}^{2}}\left(2 u_{i, j}^{n}-u_{i-1, j}^{n}-u_{i+1, j}^{n}\right)+\frac{\tau}{h_{x}^{2}}\left(2 u_{i, j}^{n}-u_{i, j-1}^{n}-u_{i, j+1}^{n}\right)=u_{i, j}^{n-1}+\tau f_{i, j}^{n-1}
$$


where $f^{n_{i}, j}$ is non-linear part which describes calcium release in model equation.

Dirichlet boundary conditions are described in a following form:

$$
u_{0, j}^{n}=u_{m_{1}, j}^{n}=u_{i, 0}^{n}=u_{i, m_{2}}^{n}=0
$$

Conjugated Gradients method is used to solve linear system based on these expressions.

\section{Results and Discussion}

We performed a series of computer experiments for the modelling of $\mathrm{Ca}^{2+}$ release process and RyRs activation taking into account $\mathrm{Ca}^{2+}$ diffusion within the dyadic space. Standard model parameters were taken from the $\mathrm{Ca}^{2+}$-dynamics model in cardiac cell [12] to compare our previous simulation results [10] with the averaged $\mathrm{Ca}^{2+}$ and buffer concentrations in the current work.

$k_{b C M}=0.542 \mathrm{~ms}^{-1}, k_{b C Q}=0.445 \mathrm{~ms}^{-1}, k_{f C M}=227.7 \mathrm{mM}^{-1} \mathrm{~ms}^{-1}, k_{f C Q}=0.534 \mathrm{mM}^{-1} \mathrm{~ms}^{-1} ; C_{t o t}=10 \mathrm{mM}: C M_{t o t}=0.045$ $\mathrm{mM} ; \mathrm{d}=10^{-10} \mathrm{~m}^{2} / \mathrm{s}, V_{j s R} / V_{s s}=1.6$.

Parameters of the computational method: number of mesh nodes: $M_{x}=M_{y}=332$; a single RyR width: $L_{R y R}=37 \mathrm{~nm}$, size of a single mesh node: $L$ mesh $=1 \mathrm{~nm}$, timestep: $\mathrm{dt}=0.01 \mathrm{~ms}$

$\mathrm{Ca}^{2+}$ concentrations initial values: $\operatorname{CajsR}(t=0)=1 \mathrm{mM}, \operatorname{Cass}(t=0)=0, N_{\text {open }}(t=0)=0$.

Electron-conformational modl parameters: $K_{c_{a}}=500 \mu \mathrm{M}$, Cass_crit $_{1}=100 \mu \mathrm{M}, \alpha=0.0012 \mathrm{~ms}^{-1} \mu \mathrm{M}^{-1}$. At the initial time all channels were closed, Cass_mesh $=150 \mu$ Mnear the central RyR.

The time course of the RyR-cluster opening is shown on the pic.2. In the beginning of the experiment a small number of RyRs transform to the opened state due to the tunneling process. Diffusion causes an increase of a local Cass concentration of the nearest neighbors, switching on calcium dependent electronic stochastic transitions, thus "domino-like" CICR process occurs during the first period of $\mathrm{Ca}^{2+}$ spark formation.

In our experiment $\mathrm{Ca}^{2+}$ release at $t=10 \mathrm{~ms}$ was sufficient to decrease $C a_{j S R}$ below the threshold level $K_{c a}=500 \mu \mathrm{M}$ (pic. 3). A reverse tunneling process from the opened to the closed state takes place, a probability of RyRs activation decreases at $t>10 \mathrm{~ms}$ because of the lumen depletion and due to $\mathrm{Ca}^{2+}$ diffusion over the borders of the dyadic space.

According to these observed effects we've made a conclusion, that in our model spark can terminate due to lumen depletion and a RyR's stochastic attrition also plays a major role.

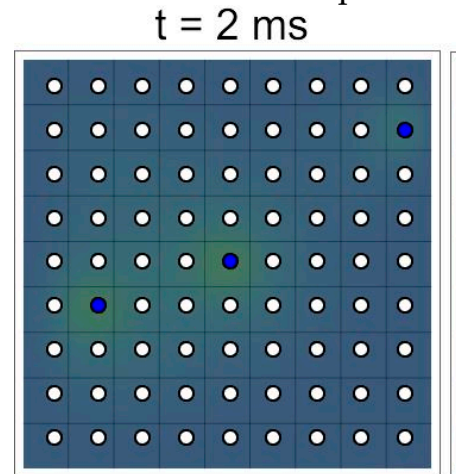

$\mathrm{t}=6 \mathrm{~ms}$

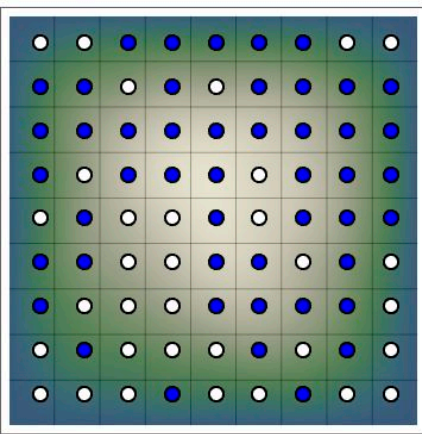

$\mathrm{t}=3 \mathrm{~ms}$

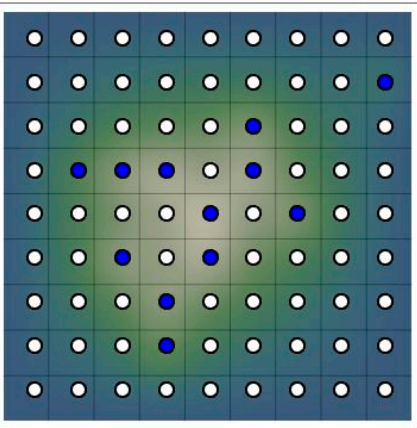

$\mathrm{t}=15 \mathrm{~ms}$

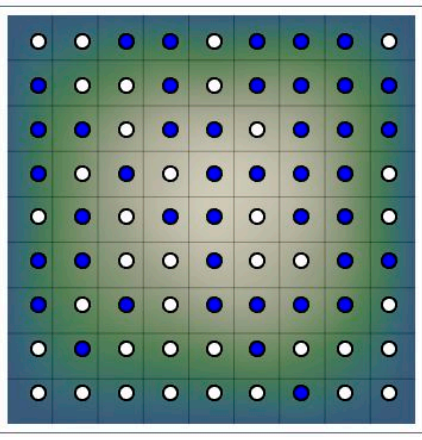

$\mathrm{t}=5 \mathrm{~ms}$

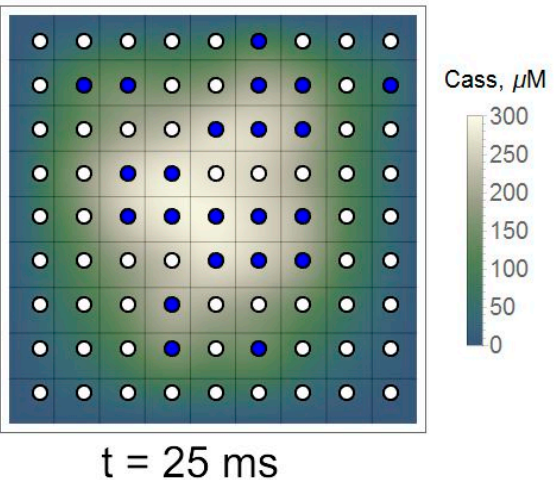

$\mathrm{t}=25 \mathrm{~ms}$

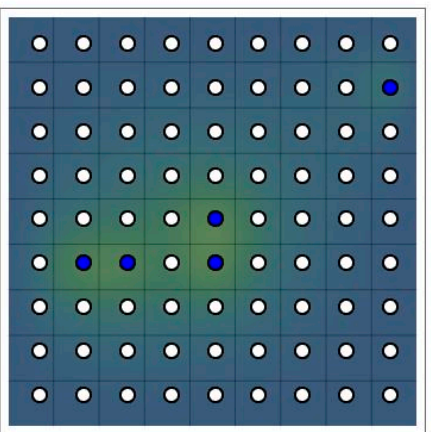


Pic. 2. Simple $\mathrm{Ca}^{2+}$ spark initiation-spread-termination process. Density plot illustrates a simulated time course of RyR's opening and spatial distribution of local $\mathrm{Ca}^{2+}$ concentrations in the dyadic space. Blue circles correspond to open RyRs, white - closed.

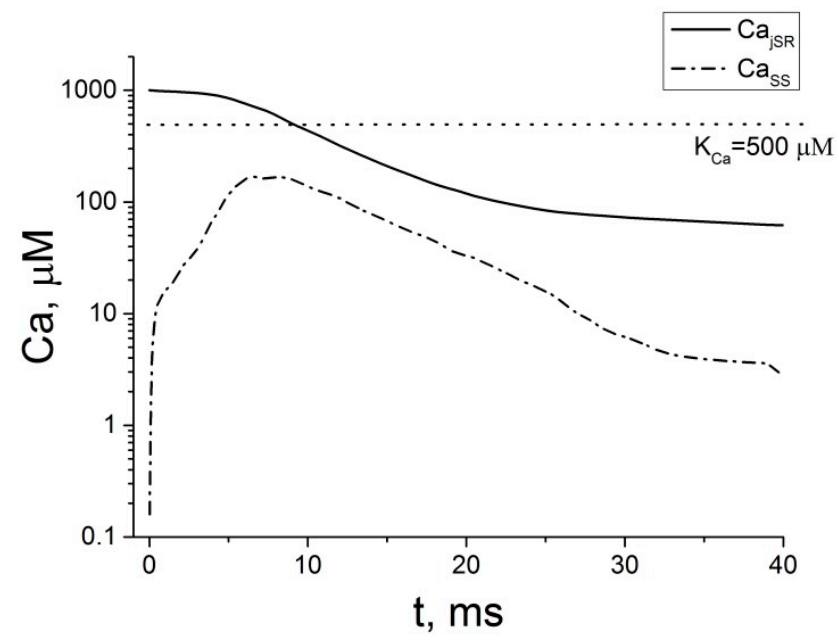

Pic. 3. Time course of calcium concentrations in the $\mathrm{Ca}^{2+}$-release unit compartments. Average $\mathrm{Ca}^{2+}$ concentration in the dyadic space (Cass) and lumen concentration $\left(C a_{j s R}\right)$ during the spark initiation-spread-termination process are shown. Dashed line corresponds to critical value of $C a_{j S R}$.

We observed a dependence of spark initiation and spread rate on the $\mathrm{Ca}^{2+}$ diffusion coefficient in the dyadic space. As one can see on the pic. 4 the increase of diffusion coefficient accelerates RyRs opening in the cluster and the amplitude of $N_{\text {open }}(t)$. As a result the lumen depletion happens earlier, thus spark termination begins earlier.

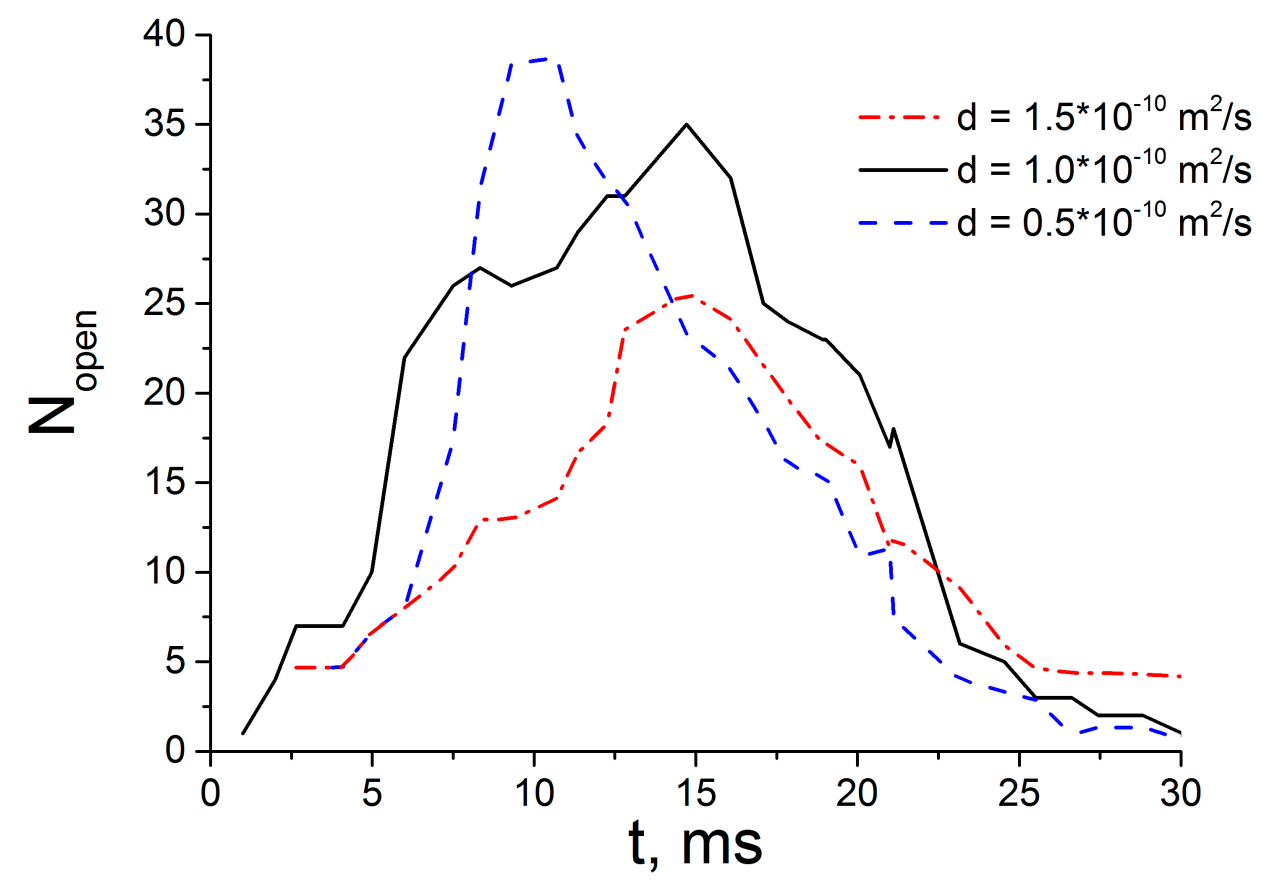

Pic. 4. Time course of the number of opened RyR-channels in the cluster during the calcium spark for different values of diffusion rate $d$.

\section{Conclusions and Perspectives}

Computer simulations within the framework of the united model of $\mathrm{Ca}^{2+}$ dynamics in $\mathrm{Ca}^{2+} \mathrm{RU}$ is able to reproduce experimental data of calcium sparks observations [] in the cardiac cells. 
Incorporation of the RyRs Electron-Conformational Model to the theory of $\mathrm{Ca}^{2+}$ dynamics in RU allows us to describe the process of RyRs activation in details on the macromolecular level but in terms of only two parameters (Kca, Cass_crit), responsible for RyRs activation by $\mathrm{Ca}^{2+}$ ions from the dyadic and from the luminal site. As a summary, we've proved in our simulations that mechanisms of RyRs stochastic attrition and the process of the lumen depletion play the major role of sparks termination.

In the nearest future we plan to take into account conformational RyR-RyR coupling which can be described in terms of the Electron-Conformational Model and to explore the contribution of this phenomenon to the process of the sparks initiation-spread-termination. Previously ECM has shown a brilliant ability to describe thermal effects of the RyR conformational dynamics [8, 9]. Thermal fluctuation terms will be added to the system of equations of the United model to observe effects of the temperature impact on the $\mathrm{Ca}^{2+}$ sparks dynamics.

\section{References}

1. Cheng, H., Lederer, W. J., \& Cannell, M. B. (1994). Calcium sparks: elementary events underlying excitation-contraction coupling in heart muscle (Doctoral dissertation, University of Maryland).

2. Soeller C., Cannell M.B. Estimation of the Sarcoplasmic Reticulum Ca ${ }^{2+}$ Release FluxUnderlying Ca ${ }^{2+}$ Sparks. Biophysical journal. 2002. V. 82. №. 5. P. 2396-2414.

3. Chen B., Wu Y., Mohler P.J., Anderson M.E., Song L.S. Local control of Ca ${ }^{2+}$-induced Ca ${ }^{2+}$ release in mouse sinoatrial node cells. Journal of molecular and cellular cardiology. 2009. V. 47. № 5. P. 706-715.

4. Cannell M.B., Kong C.H.T., Imtiaz M.S., Laver D. R. Control of sarcoplasmic reticulum Ca ${ }^{2+}$ release by stochastic RyR gating within a 3D model of the cardiac dyad and importance of induction decay for CICR termination. Biophysical journal. 2013. V. 104. № 10. P. 2149-2159.

5. Vierheller J., Neubert W., Falcke M., Gilbert S.H., Chamakuri N. A multiscale computational model of spatially resolved calcium cycling in cardiac myocytes: from detailed cleft dynamics to the whole cell concentration profiles. Frontiers in physiology. 2015. V. 6.

6. Williams G.S., Chikando A.C., Tuan H.T.M., Sobie E.A., Lederer W.J., Jafri M. S. Dynamics of calcium sparks and calcium leak in the heart. Biophysical journal, 2011. V. 101. № 6. P. 1287-1296.

7. Moskvin A.S., Ryvkin A.M., Solovyova O.E., Markhasin V.S. Electron-conformational transformations in nanoscopic RyR channels governing both the heart's contraction and beating. JETP letters. 2011. V. 93. № 7. P. 403-408.

8. Moskvin A.S., Iaparov B.I., Ryvkin A.M., Solovyova O.E. The temperature effect on cardiac ryanodine receptor gating and conductance: Mathematical modeling. Biophysics. 2016. V. 61. № 4. P. 614-621.

9. Moskvin A.S., Iaparov B.I., Ryvkin A.M., Solovyova O.E., Markhasin V.S. Electronconformational transformations govern the temperature dependence of the cardiac ryanodine receptor gating. JETP letters. 2015. V. 102. № 1. P. 62-68.

10. Ryvkin A.M., Moskvin A.S., Solovyova O.E., Markhasin V.S. Simulation of the auto-oscillatory calcium dynamics in cardiomyocytes in terms of electron conformational theory. Doklady Biological Sciences. - MAIK Nauka/Interperiodica. 2012. V. 444. №. 1. P. 162-168.

11. Hake J., Edwards A.G., Yu Z., Kekenes-Huskey P.M., Michailova A.P., McCammon J.A., Holst M.J., Hoshijima M., McCulloch A.D. Modelling cardiac calcium sparks in a three-dimensional reconstruction of a calcium release unit. The Journal of Physiology. 2012. V. 590. № 18. P. 44034422.

12. Lakatta E.G., Maltsev V.A., Vinogradova T.M. A coupled SYSTEM of intracellular Ca ${ }^{2+}$ clocks and surface membrane voltage clocks controls the timekeeping mechanism of the heart's pacemaker. Circulation research. 2010. V. 106. № 4. P. 659-673. 